\title{
A Filter-and-Refine Approach to Mine Spatiotemporal Co-occurrences
}

\author{
Karthik Ganesan Pillai \\ Computer Science Department \\ Montana State University \\ Bozeman, MT 59717, USA \\ k.ganesanpillai@cs.montana.edu
}

\author{
Rafal A. Angryk, Berkay Aydin \\ Department of Computer Science \\ Georgia State University \\ Atlanta, GA 30302-3994, USA \\ angryk@cs.gsu.edu \\ baydin2@student.gsu.edu
}

\begin{abstract}
Spatiotemporal co-occurrence patterns (STCOPs) represent the subsets of event types that occur together in both space and time. However, the discovery of STCOPs in data sets with extended spatial representations that evolve over time is computationally expensive because of the necessity to calculate interest measures to assess the co-occurrence strength, and the number of candidates for STCOPs growing exponentially with the number of spatiotemporal event types. In this paper, we introduce a novel and effective filterand-refine algorithm to efficiently find prevalent STCOPs in massive spatiotemporal data repositories with polygon shapes that move and evolve over time. We provide theoretical analysis of our approach, and follow this investigation with a practical evaluation of our algorithm effectiveness on three real-life data sets and one artificial data set.
\end{abstract}

\section{Categories and Subject Descriptors}

H.4 [Spatio-Temporal Data Analysis]: Spatial Data Mining and Knowledge Discovery; D.2.8 [Geographic Information Retrieval]: Spatial Data Structures and Algorithms

\section{General Terms}

Spatiotemporal Data Mining

\section{Keywords}

evolving spatiotemporal events; extended spatial representations; spatiotemporal co-occurrence patterns;

\section{Introduction}

The growth of data volumes in nearly all scientific disciplines, business sectors and federal agencies is reaching historic proportions [3]. The data acquisition speed is becoming progressively faster and leading to uncontrollably growing

Permission to make digital or hard copies of all or part of this work for personal or classroom use is granted without fee provided that copies are not made or distributed for profit or commercial advantage and that copies bear this notice and the full citation on the first page. Copyrights for components of this work owned by others than the author(s) must be honored. Abstracting with credit is permitted. To copy otherwise, or republish, to post on servers or to redistribute to lists, requires prior specific permission and/or a fee. Request permissions from Permissions@acm.org.

SIGSPATIAL'13 Nov 05-08 2013, Orlando, FL, USA

Copyright is held by the owner/author(s). Publication rights licensed to ACM.

ACM 978-1-4503-2521-9/13/11\$15.00.

http://dx.doi.org/10.1145/2525314.2525367 data. In astronomy in particular, rapid advances in three technology areas (telescopes, detectors and computation) have caused the generation of massive data [2]. With the launch of NASA's Solar Dynamics Observatory (SDO) mission, solar physics researchers started dealing with big data. SDO's space telescope registers approximately 70,000 high resolution $(4096 \times 4096$ pixels $)$ images daily, obtaining one image every ten seconds [16]. It sends out 0.55 petabytes of raster data to Earth each year [16]. This trend in solar data is anticipated to be sustained by ground-based Advanced Technology Solar Telescope, which is expected to capture one million images per day and generate three to five $\mathrm{PB}$ of data per year [13]. To facilitate the important needs of Space Weather monitoring (which can have vital impacts on space and air travel, power grids, GPS and communication devices), many software modules working continously on massive SDO raster data and generating object data with spatiotemporal characteristics. One application area for our research is solar events, since spatiotemporal co-occurrence patterns (STCOPs) frequently occur among various solar events. Given a spatiotemporal (abbreviated as ST in the rest of the paper) database in which data objects are represented as polygons which continuously change their movement, shape, and size, our goal is to identify STCOPs representing the subset of different event types that occur together in space and time.
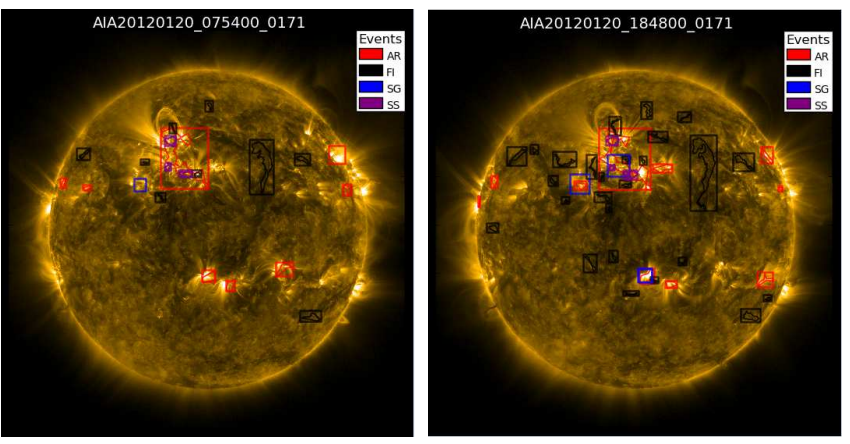

Figure 1: Images showing the evolution of four types of solar events (NASA instrument and time stamp are printed on the top of each image).

\subsection{A Real-life Example}

STCOPs frequently occur among various solar events. Fig. 1 shows four types of solar events, Active Regions (AR), Filaments (FL), Sigmoids (SG), and Sunspots (SS) in spa- 
tial and temporal contexts with their corresponding shapes and bounding boxes. As seen in Fig. 1, the shapes of the solar events are represented as extended spatial representations (polygons). Moreover, the shape, size, and location of the solar events continuously evolve over time. All of these factors influence relationships between various solar events, which lead to complex spatial and temporal interactions. Identifying STCOPs on the Sun could help us better understand the relationships between solar events and lead to better modeling and forecasting of important events such as coronal mass ejections and solar flares, which impact radiation in space, and can reduce the safety of space and air travel [11], disrupt intercontinental communication [11] and GPS [11], and even damage power grids [11].

\subsection{Contributions}

This paper presents a novel approach to our recent work initiated in [17], where we introduced the STCOPs mining problem, developed a general framework to discover STCOPs, proposed a naïve Apriori-based [1] STCOPs mining algorithm, and evaluated it using a real-life data set. This work makes the following new contributions: (1) We introduce a new and computationally efficient STCOPs mining algorithm (FastSTCOPs-Miner), using a filter-and-refine strategy to prune irrelavent STCOPs based on the usage of all-confidence $(O M A X)$ measure as filtering mechanism for Jaccard-based analysis which is the standard measure in data mining $[17,21,20]$. (2) We provide a theoretical analysis to show the correctness and completeness of our FastSTCOPs-Miner algorithm. (3) We experimentally verify the correctness of proposed algorithm with our naïve STCOPs algorithm [17] on three real-life data sets and one artificial data set, and provide extended experimental results demonstrating the computational and memory efficiency of the FastSTCOPs-Miner algorithm.

The following issues are beyond the scope of this paper: (1) Determining threshold values to capture the strength of ST neighborhood relation between the instances of different event types; (2) ST data indexing strategies related to mining these events; and (3) Comparison with non-Apriori approaches. The reason we chose to start our work with Apriori-based technique is because we believe that our filterand-refine strategy is easier to explain in these simpler settings. We hope to be able to incorporate the filter step in our future works on non-Apriori approaches; however, currently there is no non-Apriori method that is capable of taking advantage of our novel $O M A X$-based approach to filter patterns.

The rest of the paper is organized as follows: Sec. 2 gives background information on related work. We review important concepts of modeling STCOPs for evolving extended spatial representations and provide theoretical analysis of our approach in Sec. 3. In Sec. 4, we present our proposed FastSTCOPs-Miner algorithm. Finally, we present a variety of experiments demonstrating the effectiveness of our approach with real-life and artificial data sets, concluding with a summary of results and future work.

\section{Related Work}

Since ST data mining is an important area, many algorithms have been proposed in literature for co-location mining in ST databases: topological pattern mining [22], co-location episodes [4], mixed drove co-occurrence mining [5], spatial co-location pattern mining from extended spatial represen- tations [23], ST pattern mining in scientific data [24], and interval orientation patterns [15]. However, none of these approaches focus on mining ST co-occurrences from data represented as polygons evolving in time.

Mining topological patterns, also called co-location patterns, from ST databases was introduced by Wang et al. in [22]. The authors introduced a summary-structure to record the number of instances of a feature in a region for a given time window. The authors used the summary-structure to approximate the instance counts of a co-location pattern. They also introduced the TopologyMiner algorithm to discover frequent co-location patterns in a depth-first manner.

Cao et al. introduced the problem of mining co-location episodes in ST data [4]. The authors define a co-location episode as a sequence of co-location patterns with some common feature type across consecutive time slots. The authors also introduced a two-step framework for mining co-location episodes. In the first step of the framework, the authors transform the original trajectories of moving objects to a sequence of close features to the corresponding object. Eventually, the object pairs of different feature types $\left(f_{i}, f_{j}\right)$ with close concurrent subsequences are identified. In the next step of the framework, an Apriori-based [1] technique is used to discover the frequent episodes, using the transformed sequence of feature sets.

Celik et al. introduced the problem of mining mixed-drove ST co-occurrence patterns (MDCOPs) in ST data [5]. An MDCOP is defined as a subset of ST mixed feature types whose instances are neighbors in space and time. They introduced the MDCOP-Miner algorithm, which extends the standard spatial co-location mining algorithm [9] to include time information. The algorithm first discovers all size- $(k)$ spatially prevalent MDCOPs, and applies a time-prevalence based filtering to discover MDCOPs. Finally, the MDCOPMiner algorithm generates size- $(k+1)$ candidate MDCOPs using size- $(k)$ MDCOPs.

Xiong et al. introduced the problem of mining spatial colocation patterns from extended spatial representations in [23]. The authors introduced a buffer based model to find colocation patterns. In the buffer based model, the neighborhood of an extended spatial representation is defined by the spatial buffer operation. The Euclidean neighborhood $N(f)$ of some feature $f$ is defined as the union of neighborhoods for every instance $i$ of the feature $f$. The Euclidean neighborhood $N\left(f_{1}, \ldots, f_{k}\right)$ for a feature set $E=\left\{f_{1}, \ldots, f_{k}\right\}$ is defined as $\cap_{i=1}^{|E|} N\left(f_{i}\right)$ s.t $f_{i} \in E$. The authors introduced the Apriori-based EXCOM algorithm to find spatial co-location patterns in data sets with extended spatial representations.

Mining ST patterns in scientific data was first introduced by Yang et al. in [24]. They introduced a general framework to discover spatial associations and ST episodes for scientific data sets. The authors modeled features as geometric objects rather than points. They also extend their approach to accomodate emporal information and propose an algorithm to derive ST episodes.

The problem of mining interval orientation patterns in ST data was introduced by Patel in [15]. The event features are modeled by taking the duration of feature into account. Thus, the author was able to capture the temporal influence of a feature on other features within a spatial neighborhood. An Interval Orientation (IO) pattern is a frequent sequence of features with the annotations of temporal and directional 


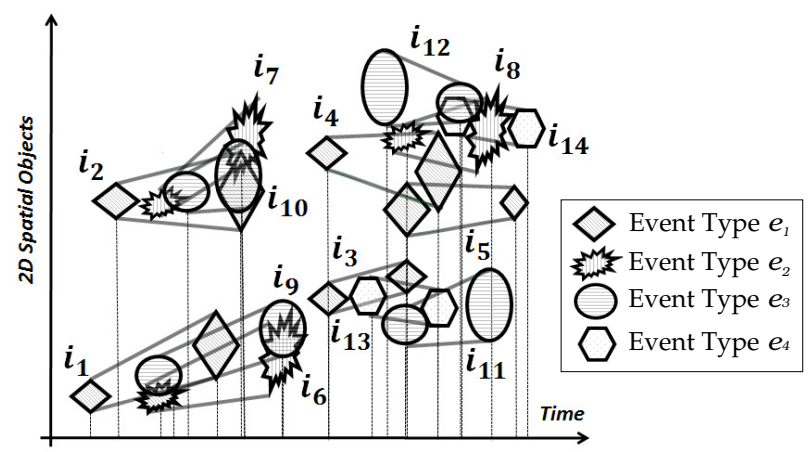

Figure 2: A ST dataset with 2D spatial objects evolving in time.

relationships between every pair of features. The author, introduced an algorithm called IOMiner to mine frequent IO patterns.

In 2012, Ganesan Pillai et al. were the first who introduced the problem of discovering STCOPs mining from data with polygon-like representations that evolve over time [17], [18].

\section{Basic Concepts \\ 3.1 Modeling STCOPs}

Given a set of ST event types denoted $E=\left\{e_{1}, \ldots, e_{M}\right\}$, and a set of continously evolving instances $I=\left\{i_{1}, \ldots, i_{N}\right\}$ of these event types, such that $M \ll N$. An STCOP is a subset of ST event types that co-occur in both space and time.

In Fig. 2, we show an example data set that we will use to explain the definitions in detail. In Table 1, we show the Instance ID, Start Time, and End Time of instances of different event types from our example data set in Fig. 2. This data set contains four event types. The event type $e_{1}$ has a total of five ST instances $\left(i_{1}-i_{5}\right), e_{2}$ has three instances $\left(i_{6}-i_{8}\right), e_{3}$ has four instances $\left(i_{9}-i_{12}\right)$, and $e_{4}$ has two instances $\left(i_{13}-i_{14}\right)$. For simplicity, in this example we do not show the sequence of $2 D$ shapes that reflect the ST evolution of our data. In our example, $E=\left\{e_{1}, e_{2}, e_{3}, e_{4}\right\}$, $M=4$, and $N=14$ (all instance IDs are listed in the first column of Table 1). From our real-life data gathered by SDO mission shown in Fig. 1, we can derive the example data set shown in Fig. 2 by following the trajectories of instances of different solar event types. In Fig. 1 time stamps are printed on top of each image showing the evolution of instances over time.

Definition 1. A size- $k$ STCOP is denoted as $S E=$

\begin{tabular}{cccc}
\hline$\underline{\text { Instance_ID Event }} \begin{array}{ccc}\text { Type } \\
i_{1}\end{array}$ & $\begin{array}{c}\text { Start Time } \\
\text { (HH:MM) }\end{array}$ & $\begin{array}{c}\text { End Time } \\
\text { (HH:MM) }\end{array}$ \\
\hline$i_{2}$ & $e_{1}$ & $10: 00$ & $10: 30$ \\
$i_{3}$ & $e_{1}$ & $10: 10$ & $10: 40$ \\
$i_{4}$ & $e_{1}$ & $11: 00$ & $11: 20$ \\
$i_{5}$ & $e_{1}$ & $11: 20$ & $11: 30$ \\
$i_{6}$ & $e_{2}$ & $10: 20$ & $11: 50$ \\
$i_{7}$ & $e_{2}$ & $10: 20$ & $10: 50$ \\
$i_{8}$ & $e_{2}$ & $11: 20$ & $11: 40$ \\
$i_{9}$ & $e_{3}$ & $10: 20$ & $10: 50$ \\
$i_{10}$ & $e_{3}$ & $10: 30$ & $10: 40$ \\
$i_{11}$ & $e_{3}$ & $11: 20$ & $11: 40$ \\
$i_{12}$ & $e_{3}$ & $11: 10$ & $11: 30$ \\
$i_{13}$ & $e_{4}$ & $11: 10$ & $11: 30$ \\
$i_{14}$ & $e_{4}$ & $11: 30$ & $12: 00$ \\
\hline
\end{tabular}

Table 1: Temporal information about event instances of data shown in Fig. 2 $\left\{e_{1}, \ldots, e_{k}\right\}$, where $S E \subseteq E, S E \neq \emptyset$ and $1<k \leq M$.

We can have multiple size- $k$ STCOPs derived from the set $E$, so to separate them we will use subscripts in future definitions, (e.g., $S E_{i}$ ) with an arbitrarily chosen subscripts to denote uniqueness, i.e. $S E_{i} \neq S E_{j}$, and $S E_{i}$ and $S E_{j}$ contain different event types. Note that indices $(i$ or $j$ ) do not indicate number of event types involved in the cooccurrence (we only use $k$ for that).

Definition 2. pat_instance is a pattern instance of an STCOP $S E_{i}$, if and only if pat_instance contains unique instances of all event types from $S E_{i}$. No proper subset of pat_instance is considered to be a pattern instance of $S E_{i}$.

For example, $\left\{i_{2}, i_{7}, i_{10}\right\}$ is a size-3 $(k=3)$ pattern instance of co-occurrence $S E_{i}=\left\{e_{1}, e_{2}, e_{3}\right\}$ in the example ST data set.

Definition 3. A collection of pattern instances of $S E_{i}$ is a table instance of $S E_{i}$, and is denoted as tab_ins $\left(S E_{i}\right)$.

For example, $\left\{\left\{i_{1}, i_{6}, i_{9}\right\},\left\{i_{2}, i_{7}, i_{10}\right\}\right\}$ is a size-3 $(k=3)$ tab_ins $\left(S E_{i}=\left\{e_{1}, e_{2}, e_{3}\right\}\right)$ in the example data set presented in Fig. 2 and Table 1.

Definition 4. A prevalent STCOP is of the form $S E_{i}(c c e, p)$, where $S E_{i}$ is a $\mathrm{ST}$ co-occurrence pattern, and parameters cce, $p$ characterize the prevalent pattern in the following manner:

1. cce stands for co-occurrence coefficient [17]. It is an indicator of the strength of a ST relation's occurrence that is investigated. The naïve STCOPs algorithm uses the ST relation Overlap for cce. To distinguish the ST relation from the purely spatial one, we will use capital letter in the name of the former. Some examples of ST Overlap are $\left\{i_{1}, i_{6}\right\},\left\{i_{2}, i_{7}\right\}$, and $\left\{i_{7}, i_{10}\right\}$ in Fig. 2. We will discuss this in more detail in Sec. 3 3.

2. $p$ is the prevalence measure. The prevalence measure emphasizes how interesting the ST co-occurrences are based on prevalence. In our investigation, we used the participation index $(p i)$, proposed in [9], as the prevalence measure. The participation index is monotonically non-increasing when the size of the STCOP increases, which is exploited for computational efficiency [9].

\subsection{Measures}

Our naïve STCOPs algorithm [17] uses the ST co-occurrence coefficient to calculate cce. The ST co-occurrence coefficient is closely related to the coefficient of areal correspondence (CAC) proposed in [21]. CAC is usually computed for any two (or more, for longer patterns) overlapping polygons as the area of intersection, divided by the area of union (i.e. spatial version of Jaccard measure). In our work [17], we extend CAC to three dimensions (two dimensions correspond to space and the third dimension corresponds to time) and calculate the ST co-occurrence coefficient using ST volumes: (1) the volume of Intersection of two or more ST objects' trajectories, and (2) the volume of the Union of their trajectories.

Definition 5. The Intersection volume of a size- $k$ pattern instance, denoted $V\left(i_{1} \cap i_{2}, \ldots, i_{k-1} \cap i_{k}\right)$, is the volume of the three dimensional object representing the Intersection of the trajectories of all instances involved in a given pattern instance.

Definition 6. The Union volume of a size- $k$ pattern instance, denoted as $V\left(i_{1} \cup i_{2}, \ldots, i_{k-1} \cup i_{k}\right)$, is the volume of 


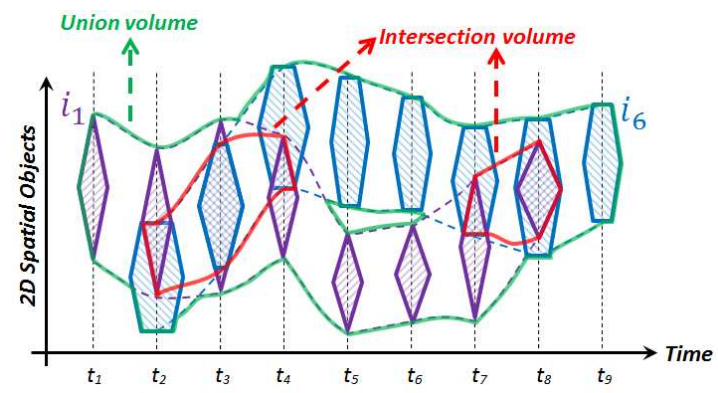

Figure 3: Example of size-2 co-occurrence of ST objects (assume $i_{1}$ is an instance of $S T$ event type $e_{1}$, and $i_{6}$ is an instance of $S T$ event type $\left.e_{2}\right)$. Color red reflects $S T$ Intersection volume, while color green represents ST Union volume.

the three dimensional object representing the Union of the trajectories of all instances involved in a given co-occurrence.

\subsection{Co-occurrence coefficient $c c e$}

We use the ST co-occurrence coefficient (cce) to assess the strength of the ST relation Overlap. cce is typically calculated for a size- $k$ pattern instance as the ratio $J=$ $\frac{V\left(i_{1} \cap i_{2}, \ldots, i_{k-1} \cap i_{k}\right)}{V\left(i_{1} \cup i_{2}, \ldots, i_{k-1} \cup i_{k}\right)}$. The symbol $J$ stands for the Jaccard measure, which is commonly accepted by data mining practitioners to measure the co-occurence of items in shopping baskets [12, 20], among spatial objects [21], and in ST data [17]. Computing the cce for extended ST representations such as evolving polygons is not a trivial task for massive data sets. In Fig. 3, we show the movement of a pair of instances of two different event types that change sizes, shapes and locations and movements and directions through time instances. We also show the regions of intersection and the regions of spatial unions at different time instances. Moreover, the volumes resulting from the Intersection and Union of objects' trajectories are shown in Fig. 3. If we assume that instances $\left\{i_{1}, i_{6}\right\}$, in our example data set (Fig. 2 and Table 1), have ST Intersection volume $V\left(i_{1} \cap i_{6}\right)=241$ and a ST Union volume $V\left(i_{1} \cup i_{6}\right)=1005$, then, the ST cooccurrence coefficient is equal to $\frac{V\left(i_{1} \cap i_{6}\right)}{V\left(i_{1} \cup i_{6}\right)}=0.23$ (see the notes under Table 2 for detailed calculation of cce). In Table 2 , the third column shows time instances (with $\Delta t=10$ min. used as our sampling interval), the fourth column Area $\left(i_{1} \cap i_{6}\right)$ shows intersection areas, and the fifth column Area $\left(i_{1} \cup i_{6}\right)$ shows union areas at each time instant.

\begin{tabular}{|c|c|c|c|c|}
\hline $\begin{array}{c}\text { Instance of } \\
e_{1}\end{array}$ & $\begin{array}{c}\text { Instance of } \\
e_{2}\end{array}$ & $\begin{array}{l}\text { Time } \\
\text { Instant } \\
(\Delta t=10 \\
\text { minutes })\end{array}$ & Area $\left(i_{1} \cap i_{6}\right)$ & $\operatorname{Area}\left(i_{1} \cup i_{6}\right)$ \\
\hline$i_{1}$ & $i_{6}$ & $t_{1}=10: 00$ & 0 & 60 \\
\hline$i_{1}$ & $i_{6}$ & $t_{2}=10: 10$ & 25 & 120 \\
\hline$i_{1}$ & $i_{6}$ & $t_{3}=10: 20$ & 95 & 115 \\
\hline$i_{1}$ & $i_{6}$ & $t_{4}=10: 30$ & 15 & 140 \\
\hline$i_{1}$ & $i_{6}$ & $t_{5}=10: 40$ & 0 & 150 \\
\hline$i_{1}$ & $i_{6}$ & $t_{6}=10: 50$ & 0 & 140 \\
\hline$i_{1}$ & $i_{6}$ & $t_{7}=11: 00$ & 16 & 130 \\
\hline$i_{1}$ & $i_{6}$ & $t_{8}=11: 10$ & 90 & 90 \\
\hline$i_{1}$ & $i_{6}$ & $t_{9}=11: 20$ & 0 & 60 \\
\hline
\end{tabular}

Table 2: Example of a pattern instance in tab_ins of $S E_{i}=$ $\left\{e_{1}, e_{2}\right\}$ with calculation of cce from data shown in Fig. 3.

We would like to point out here that computing $J$ for ST pattern instances is quite expensive (due to the necessary calculations of intersection and union geometries for each time stamp, and storage space required to save these geometries). In this work, we introduce an alternate measure $O M A X$, defined as $\frac{V\left(i_{1} \cap i_{2}, \ldots, i_{k-1} \cap i_{k}\right)}{\max \left(V\left(i_{1}\right), \ldots, V\left(i_{k}\right)\right)}$, that can be effectively used to assess the ST co-occurrence strength of a pattern instance, and it will provide significant speed-up for discovery of STCOPs based on the commonly used Jaccard measure. $O M A X$ is the foundation of our filter-and-refine approach. We filter out the pattern instances that do not satisfy the user-specified threshold, $c_{\text {th }}$, with $O M A X$ (in Sec. 3.6 we will prove that such patterns can not satisfy $J$ with the same $c c e_{t h}$ as well), and then calculate $J$ for the reduced set of pattern instances. OMAX represents the all-confidence measure [14] in classical association rules mining literature and it is time and storage-wise significantly cheaper to calculate than Jaccard on ST data. We will show the proofs for the completeness of STCOPs generated with our filter-and-refine approach as well as experimental results confirming our theoretical investigations and space and time scalability of our approach through the rest of this paper.

\subsection{Prevalence of STCOPs}

Definition 7. The participation index $p i\left(S E_{i}\right)$ of a ST co-occurrence $S E_{i}$ is defined as:

$$
p i\left(S E_{i}\right)=\min _{j=1}^{k} \operatorname{pr}\left(S E_{i}, e_{j}\right)
$$

where $k$ is the size of the pattern (i.e. cardinality of $S E_{i}$ ), and the participation ratio, $\operatorname{pr}\left(S E_{i}, e_{j}\right)$, for a $\mathrm{ST}$ event type $e_{j}$ is the fraction of the total number of instances of $e_{j}$ forming ST co-occurring instances in $S E_{i}$.

For example, from Fig. 2 and Table 1 we can see that the pattern instances of $S E_{i}=\left\{e_{1}, e_{2}, e_{3}\right\}$ are $\left\{\left\{i_{1}, i_{6}, i_{9}\right\},\left\{i_{2}, i_{7}, i_{10}\right\}\right\}$. Only two $\left(i_{1}, i_{2}\right)$ out of five instances of the event type $e_{1}$ participate in $S E_{i}=$ $\left\{e_{1}, e_{2}, e_{3}\right\}$. So, $\operatorname{pr}\left(\left\{e_{1}, e_{2}, e_{3}\right\}, e_{1}\right)=2 / 5=0.40$. Similarly $\operatorname{pr}\left(\left\{e_{1}, e_{2}, e_{3}\right\}, e_{2}\right)=2 / 3=0.67$, and $\operatorname{pr}\left(\left\{e_{1}, e_{2}, e_{3}\right\}, e_{3}\right)=$ $2 / 4=0.50$. Therefore the participation index of ST co-occurrence $S E_{i}=\left\{e_{1}, e_{2}, e_{3}\right\}$ is $p i\left(\left\{e_{1}, e_{2}, e_{3}\right\}\right)=$ $\min (0.40,0.67,0.50)=0.40$.

Definition 8. The ST co-occurrence $S E_{i}$ is a prevalent pattern if it satisfies a user-specified minimum participation index threshold denoted as $p i_{t h}$.

In our example above, if the minimum threshold is set to $p i_{\text {th }}=0.3$, then the $\mathrm{ST}$ co-occurrence pattern $S E_{i}=$ $\left\{e_{1}, e_{2}, e_{3}\right\}$ is a prevalent pattern.

\subsection{Problem Statement}

\section{Input:}

1. A set of ST event types $E=\left\{e_{1}, e_{2}, \ldots, e_{M}\right\}$ over a common ST framework.

2. A set of $N$ ST event instances $I=\left\{i_{1}, i_{2}, \ldots, i_{N}\right\}$, where each $i_{j} \in I$ is a tuple <instance-id, ST event type, sequence of $\angle 2 D$ shape, matching time instant $>$ pairs $>$, where the sequence of $2 D$ shape and matching time instant pairs reflects the evolution of the given ST event.

3. A user-specified ST co-occurrence coefficient threshold $\left(c c e_{t h}\right)$.

4. A user-specified participation index threshold $\left(p i_{t h}\right)$.

5. A time interval of data sampling $(\Delta t)$. All events are sampled with the same interval making the shapes of individual events exactly aligned in time.

Objective/Output: Find the complete and correct result set of spatiotemporal co-occurrence patterns (STCOPs) with $c c e>c c e_{t h}$ and $p i>p i_{t h}$. 


\subsection{Analysis of relations between $J$ and $O M A X$ measures}

In this section, we will show that we can use $O M A X$ as filter cce measure to prune candidates for $J$ measure, and reduce memory cost and running time of our $J$-based Apriori algorithm [17]. For this filter step with $O M A X$ for the $J$-based Apriori algorithm to be correct the following properties between $J$ and $O M A X$ are necessary: (1) We show that the cce values computed using the $J$ and $O M A X$ are monotonically non-increasing as the size of the pattern instance increases for a fixed $c c e_{t h}$ value (see Lemmas 3.1 and 3.2 below). (2) We show the ordering relation on the selectivity of $J$ and $O M A X$ (shown in Lemma 3.3 below). (3) We show that the STCOPs found using $J$ is a subset of the STCOPs found with $O M A X$ for a fixed $c c e_{t h}$ and $p i_{t h}$ values (see Lemma 3.4 below). All three properties are useful and necessary to reduce the number of candidate STCOPs in an accurate filter-and-refine strategy. This will greatly improve the performance of our naïve STCOPs algorithm [17].

Lemma 3.1 : The measure $J$ is anti-monotone (monotonically non-increasing) as the size of a pattern instance increases.

Proof: The measure $J$ for a size- $k$ pattern instance is defined as:

$$
\frac{V\left(i_{1} \cap i_{2}, \ldots, i_{k-1} \cap i_{k}\right)}{V\left(i_{1} \cup i_{2}, \ldots, i_{k-1} \cup i_{k}\right)}
$$

For any size- $(k+1)$ pattern instance denoted as pat_instance ${ }^{\prime}$ is equal to pat_instance $\cup\left(i_{k+1}\right)$, where pat_instance is a size- $k$ pattern instance and $i_{k+1} \notin$ pat_instance. We claim the measure $J$ follows the relation:

$$
\frac{V\left(i_{1} \cap i_{2}, \ldots, \cap i_{k}\right)}{V\left(i_{1} \cup i_{2}, \ldots, \cup i_{k}\right)} \geq \frac{V\left(i_{1} \cap i_{2}, \ldots, \cap i_{k} \cap i_{k+1}\right)}{V\left(i_{1} \cup i_{2}, \ldots, \cup i_{k} \cup i_{k+1}\right)}
$$

Therefore, we need to prove:

$$
V\left(i_{1} \cap i_{2}, \ldots, \cap i_{k}\right) \geq V\left(i_{1} \cap i_{2}, \ldots, \cap i_{k} \cap i_{k+1}\right),
$$

and

$$
V\left(i_{1} \cup i_{2}, \ldots, \cup i_{k}\right) \leq V\left(i_{1} \cup i_{2}, \ldots, \cup i_{k} \cup i_{k+1}\right)
$$

Since, adding one more instance of a different event type to a pattern instance can either reduce or not affect the volume of Intersection of instance trajectories, we obtain the relation $V\left(i_{1} \cap i_{2}, \ldots, \cap i_{k}\right) \geq V\left(i_{1} \cap i_{2}, \ldots, i_{k-1} \cap i_{k} \cap i_{k+1}\right)$ from Eq. 4. Similarly, adding one more instance of different event type to a pattern instance can either increase or not affect the volume of Union of instance trajectories, we obtain the relation $V\left(i_{1} \cup i_{2}, \ldots, i_{k-1} \cup i_{k}\right) \leq V\left(i_{1} \cup i_{2}, \ldots, i_{k-1} \cup i_{k} \cup\right.$ $\left.i_{k+1}\right)$ in Eq. 5. Thus, our relation in Eq. 3 holds for all positive real numbers that represent volumes of ST objects with evolving polygons

Lemma 3.2 : The measure $O M A X$ is anti-monotone (monotonically non-increasing) as the size of the pattern instance increases.

Proof: The measure $O M A X$ for a size- $k$ pattern instance is defined as:

$$
\frac{V\left(i_{1} \cap i_{2}, \ldots, i_{k-1} \cap i_{k}\right)}{\max \left(V\left(i_{1}\right), \ldots, V\left(i_{k}\right)\right)}
$$

For any size- $(k+1)$ pattern instance denoted pat_instance is equal to pat_instance $\cup\left(i_{k+1}\right)$, where pat_instance is a size- $k$ pattern instance and $i_{k+1} \notin$ pat_instance. We claim the measure $O M A X$ follows the relation:

$$
\frac{V\left(i_{1} \cap i_{2}, \ldots, \cap i_{k}\right)}{\max \left(V\left(i_{1}\right), \ldots, V\left(i_{k}\right)\right)} \geq \frac{V\left(i_{1} \cap i_{2}, \ldots, \cap i_{k+1}\right)}{\max \left(V\left(i_{1}\right), \ldots, V\left(i_{k+1}\right)\right)}
$$

Therefore, we need to prove:

$$
V\left(i_{1} \cap i_{2}, \ldots, \cap i_{k}\right) \geq V\left(i_{1} \cap i_{2}, \ldots, \cap i_{k} \cap i_{k+1}\right)
$$

and

$$
\max \left(V\left(i_{1}\right), \ldots, V\left(i_{k}\right)\right) \leq \max \left(V\left(i_{1}\right), \ldots, V\left(i_{k+1}\right)\right)
$$

Since, once again, adding one more instance of different event type to a pattern instance can either reduce or not affect the volume of the Intersection of the instance trajectories, we obtain the relation shown in Eq. 8. Similarly, adding another instance of a different event type to a pattern instance can not reduce the maximum volume of instance trajectories, so we obtain the relation $\max \left(V\left(i_{1}\right), \ldots, V\left(i_{k}\right)\right) \leq$ $\max \left(V\left(i_{1}\right), \ldots, V\left(i_{k}\right), V\left(i_{k+1}\right)\right)$. Thus, Eq. 7 holds for all positive real numbers that represent volumes of ST objects with evolving polygons

Lemma 3.3: The selectivity of the measures $J$ and $O M A X$ for a size-k pattern instance follows the order $\frac{V\left(i_{1} \cap i_{2}, \ldots, i_{k-1} \cap i_{k}\right)}{V\left(i_{1} \cup i_{2}, \ldots, i_{k-1} \cup i_{k}\right)} \leq \frac{V\left(i_{1} \cap i_{2}, \ldots, i_{k-1} \cap i_{k}\right)}{\max \left(V\left(i_{1}\right), \ldots, V\left(i_{k}\right)\right)}, \forall V \in R^{+}$for $k \geq 2$.

Proof: Since numerators are same, for the ordering relation $J \leq O M A X$, we can derive relations between both denominators:

$$
\max \left(V\left(i_{1}\right), \ldots, V\left(i_{k}\right)\right) \leq V\left(i_{1} \cup i_{2}, \ldots, i_{k-1} \cup i_{k}\right)
$$

Maximum volume of all trajectories is always less than or equal to the volume of union of all of them, thus the relation $J \leq O M A X$ always holds for positive real numbers

Lemma 3.4 : For a given user-specified participation index threshold $p i_{t h}$ and ST co-occurrence strength threshold $c c e_{t h}$, the set of STCOPs generated using $J$, is a subset of STCOPs generated using $O M A X$ measure, for the same $c c e_{t h}$ and $p i_{t h}$.

Proof: From Lemma 3.1 and 3.2, we know that the measures $J$ and $O M A X$ are anti-monotonic as the size of the pattern increases. Also, from Lemma 3.3, we know that ordering $J \leq O M A X$ holds.

For given user-specified thresholds $c c e_{t h}$ and $p i_{t h}$, we represent the set of all STCOPs generated for $J$ as $S T C O P_{J}$, and the set of all STCOPs generated for $O M A X$ as $S T C O P_{O M A X}$. Furthermore, we denote participation index $p i\left(S E_{i}\right)$ of a ST co-occurrence $S E_{i}$ (see Def. 7), derived by using $J$ as $p i_{J}\left(S E_{i}\right)$, and utilizing measure $O M A X$ as pi MAX $_{O}\left(S E_{i}\right)$. From Lemma 3.3, we know that the number of pattern instances found for a ST co-occurrence $S E_{i}$ follows the order $J \leq O M A X$, thus we get,

$$
\min _{j=1}^{k} \operatorname{pr}_{J}\left(S E_{i}, e_{j}\right) \leq \min _{j=1}^{k} \operatorname{prOMAX}_{\left.O E_{i}, e_{j}\right)}
$$

$$
p i_{J}\left(S E_{i}\right) \leq p i_{O M A X}\left(S E_{i}\right)
$$

Since participation index is anti-monotonic as the size of the pattern increases [9], and from Lemmas $\mathbf{3 . 1}$ and $\mathbf{3 . 2}$, and from Eq. 12, we get $S T C O P_{J} \subseteq S T C O P_{O M A X} \square$. 


\section{FastSTCOPs-Miner Algorithm}

In this section, we introduce the FastSTCOPs-Miner algorithm, which is more efficient than our naïve STCOPs algorithm [17] in the context of needed memory as well as the execution time while leading to exactly same results. This is because we apply a filter-and-refine strategy in each iteration of the algorithm. The FastSTCOPs-Miner algorithm exploits the containment relation between the STCOPs generated using Jaccard $(J)$ and $O M A X$ measures (see Sec. 3.6), to filter out candidate patterns that can not form STCOPs with the $J$.

The FastSTCOPs-Miner algorithm first filters STCOPs with $O M A X$, and then uses these filtered STCOPs to find the refined prevalent STCOPs with our standard measure, that is Jaccard $(J)$. These refined prevalent STCOPs, like in all Apriori algorithms [1], are used to generate candidate STCOPs in the next iteration of the algorithm. Thus, the FastSTCOPs-Miner algorithm continously uses a filter-andrefine strategy at each iteration of the algorithm to generate prevalent STCOPs. Even though such duplication of efforts may seem unnecessary, in Sec. 5. we will experimentally show on multitude of real-life and artificial data sets, the impressive effectiveness of our filter-and-refine strategy on ST data with evolving regions. We will also provide detailed explanation for this effectiveness.

Next, we give the pseudocode of the proposed FastSTCOPs-Miner algorithm (see Fig. 4), and explain the algorithm with a running example using the data set already shown in Fig. 2 and Table 1.

For our FastSTCOPs-Miner algorithm shown in Fig. 4, the inputs and outputs are defined as in Sec. 3.5. Steps 1 and 2 of proposed algorithm intialize the data parameters and data structures, steps 3 through 11 give an iterative process to discover the STCOPs of size greater than two. Steps 3 through 11 continue until there are no candidate STCOPs to be discovered as shown by loop condition in step 3. Step 12 returns the union of all prevalent STCOPs (patterns of all sizes). The explanations of functions in the algorithm are:

Generation of table instances of size-1 (step 2). In this function, argument $\Delta t$ represents the size of increment in time. The evolution of instances of our ST events from their birth (start) time is registered using $\Delta t$ as our time sampling frequency. The combination of the event instance ID and time step allows us to identify the appropriate spatial representation of an event at the particular moment. For example, Fig. 5 (a) shows the key columns of table instances of size-1 for our sample ST data set (Fig. 2 and Table 1). Here, the $\Delta t$ value was set to 10 minutes. The column denoted tab_ins $\left(e_{1}\right)$ represents the table instance of size-1 for event type $e_{1}$. Similarly, the columns denoted by tab_ins $\left(e_{2}\right), t a b \_i n s\left(e_{3}\right)$, and tab_ins $\left(e_{4}\right)$ represent the table instances of size- 1 for event types $e_{2}, e_{3}$, and $e_{4}$. The geometric shapes of instances in each of the presented time instances are not shown in Fig. 5 (a) for simplicity.

Generation of candidate co-occurrence patterns (step 4). This function uses an Apriori-based approach to generate candidates of size- $(k+1)$ using size- $k$ refined prevalent STCOPs (i.e. our $P R_{J k}$ in Fig. 4). However, for $k=1$ this function uses ST event types to generate candidates of size-2 (i.e. $P R_{J 1}=E$ from step 1 in Fig. 4).

Generation of filtered table instances of size- $(k+1)$

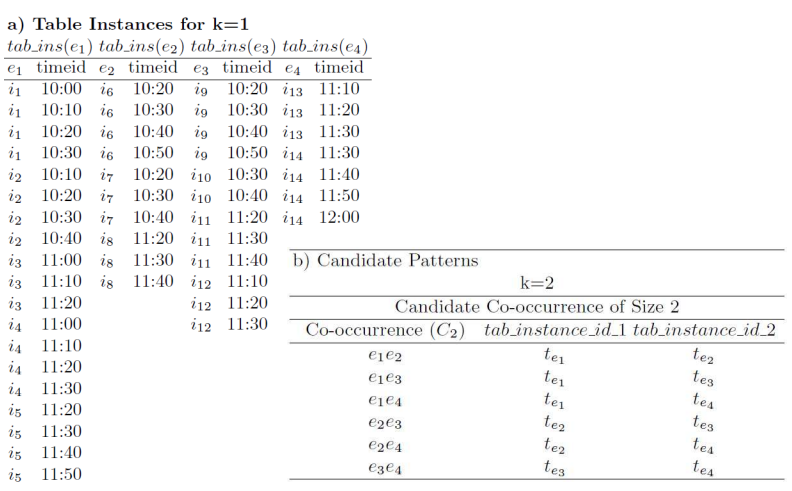

Figure 5: (a) Table Instances of size-1 and (b) Candidate Patterns for size-2 STCOPs.

(step 5). This function generates table instances for candidate patterns of size- $(k+1)$. Pattern instances for each table instance can be generated by a ST join query. The geometric shapes of the instances at each time step are saved, as these geometric shapes will be used for finding the cce of STCOPs of size three or more. In this function, we calculate the cce for each pattern instance by using $O M A X$. Pattern instances that have a cce below the user-specified $c c e_{t h}$ value are deleted from the table instance, since we know from proofs in Sec. 3.6 that they also cannot satisfy $c e_{t h}$ requirement for $J$ measure.

For example, Fig. 6 (c) shows the important columns of size-2 filtered table instances for our sample ST data set from Fig. 2 and Table 1 . The column denoted by tab_ins $\left(e_{1} e_{2}\right)$ represents the table instance of size-2 cooccurrence of event types $e_{1}$ and $e_{2}$. Similarly, the other columns represent the table instance of different event types. We also show the pattern instances that satisfy the threshold $c c e_{t h}=0.01$ calculated using $O M A X$. Moreover, we only show the key columns of table instances for simplicity. For example, in the table instance tab_ins $\left(e_{1} e_{2}\right)$ shown in Fig. 6 (c), the rows $i_{1}, i_{6}, 10: 00$ through $i_{1}, i_{6}, 10: 50$ represent a pattern instance that satisfies the threshold $c^{c} e_{t h}=0.01$. As another example, in Fig. 7 (f) we show the filtered table instances generated from candidate patterns of size $k=3$.

Generation of filtered prevalent patterns size$(k+1)$ (step 6). This function discovers filtered size- $(k+1)$ STCOPs by pruning candidate patterns in $C R_{J(k+1)}$ that have $p i<p i_{t h}$

For example, we show the $p i$ value (See Def. 7 ) at the end of each table instance in Fig. 6 (c). As seen from the Fig. 6 (c), the patterns $S E_{i}=\left\{e_{1}, e_{4}\right\}$, and $S E_{j}=\left\{e_{2}, e_{4}\right\}$ will be pruned if a value of 0.40 is set to $p i_{t h}$. Thus, the patterns that satisfy the $p i_{t h}=0.40$ are $\left\{\left\{e_{1}, e_{2}\right\},\left\{e_{1}, e_{3}\right\},\left\{e_{2}, e_{3}\right\},\left\{e_{3}, e_{4}\right\}\right\}$.

As another example, we show the $p i$ value (See Def. 7) at end of table instance tab_ins $\left(e_{1} e_{2} e_{3}\right)$ in Fig. 7 (f). As seen from the Fig. $7(\mathrm{f})$, the pattern $S E_{i}=\left\{e_{1} e_{2}, e_{3}\right\}$ is a prevalent pattern if a value of 0.40 is set to $p i_{t h}$.

Generation of refined table instances of size- $(k+1)$ (step 7). This function generates table instances for filtered prevalent STCOPs of size- $(k+1)$. Pattern instances for each table instance can be generated by using the table instances of step 5; however, additionally this function also generates and saves the Union geometries at each time step of the pattern instance. We calculate the cce for each pattern instance by using $J$ measure. Pattern instances that 
Inputs :

See Sec. 35.

Variables :

(1) $k$ the co-occurrence size (Def. 1).

(2) $C R_{J k}$ : the set of candidates for size- $(k)$ STCOPs derived from size- $(k-1)$ refined prevalent STCOPs.

(3) $T_{O M A X k}$ : a set of filtered instances of size- $(k)$ ST co-occurrences.

(4) $T R_{J k}$ : a set of refined instances of size- $(k)$ ST co-occurrences (see Def. 3).

(5) $P_{O M A X k}$ : the set of size- $k$ filtered STCOPs derived from size- $k$ candidate STCOPs.

(6) $P R_{J k}$ : the set of size- $k$ refined prevalent STCOPs derived from size- $k$ filtered STCOPs (see Def. 8).

(7) $P R_{\text {final }}$ : the union of all refined prevalent STCOPs (patterns of all sizes). // This is the final Jaccard-based prevalent patterns. Algorithm :

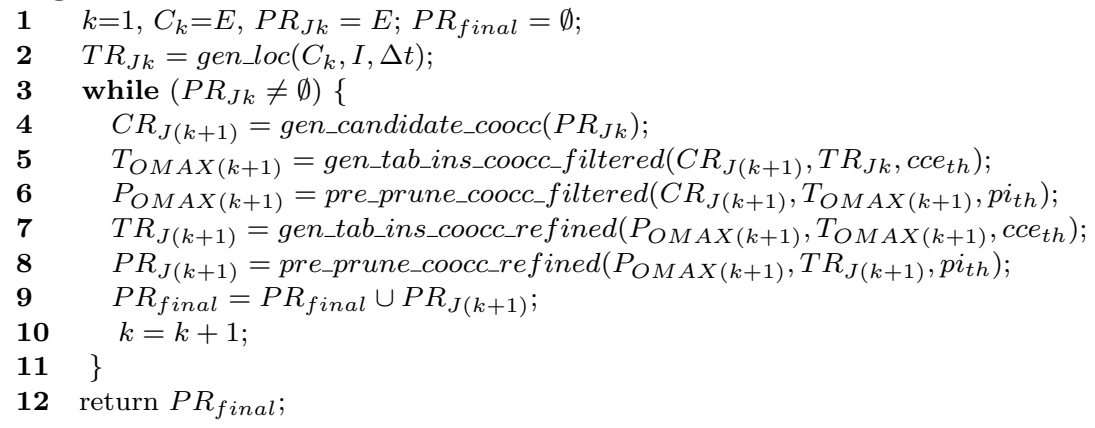

Figure 4: FastSTCOPs-Miner Algorithm

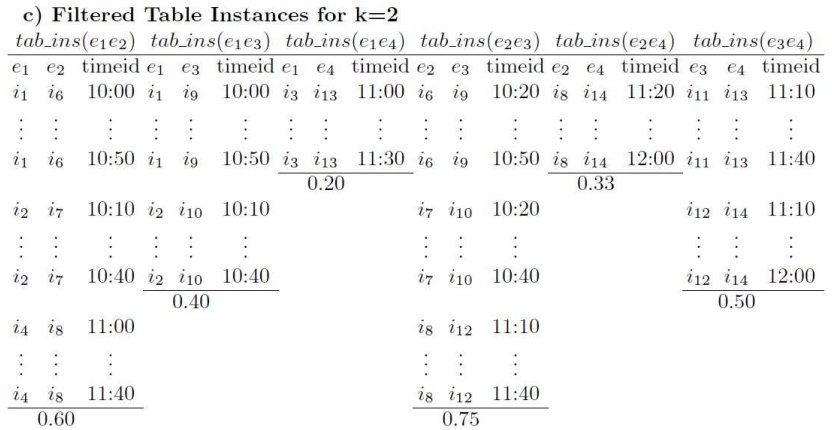

d) Refinded Table Instances for $\mathrm{k}=2$ tab_ins $\left(e_{1} e_{2}\right)$ tab_ins $\left(e_{1} e_{3}\right)$ tab_ins $\left(e_{2} e_{3}\right)$ tab_ins $\left(e_{3} e_{4}\right)$

$e_{1} e_{2}$ timeid $e_{1} \quad e_{3}$ timeid $e_{2} \quad e_{3}$ timeid $e_{3} \quad e_{4}$ timeid

$\begin{array}{lllllllllllll}i_{1} & i_{6} & 10: 00 & i_{1} & i_{9} & 10: 00 & i_{6} & i_{9} & 10: 20 & i_{11} & i_{13} & 11: 10\end{array}$

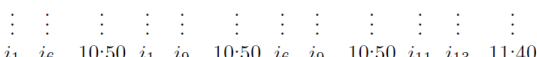

$\begin{array}{lllllllllll}i_{1} & i_{6} & 10: 50 & i_{1} & i_{9} & 10: 50 & i_{6} & i_{9} & 10: 50 & \frac{i_{11}}{i_{13}} & 11: 40 \\ i_{2} & i_{7} & 10: 10 & i_{2} & i_{10} & 10: 10 & & & & \\ 0.25 & \end{array}$

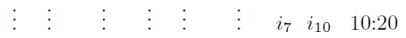

$\begin{array}{llllll}i_{2} & i_{7} & 10: 40 & i_{2} & i_{10} & 10: 40\end{array}$

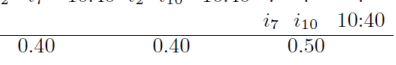

Figure 6: (c) Filtered (TOMAX2) and (d) Refined $\left(T R_{J 2}\right)$ Table Instances of size-2.

have $c c e$ less than the user-specified $c c e_{t h}$ value are deleted from the table instance.

For example, in Fig. 6 (d) we show the refined table instances generated from the refined prevalent patterns obtained in step 6 for a $p i_{t h}$ value of 0.40 . In each of the table instances shown in Fig. 6 (d), we show the key columns of pattern instances that satisfy the threshold $c c e_{t h}=0.01$ value calculated using the $J$ measure. For example, for the table instance $t a b \_i n s\left(e_{1} e_{2}\right)$, the pattern instances that satisfy the $c c e_{t h}=0.01$ for $J$ are $\left\{\left\{i_{1}, i_{6}\right\},\left\{i_{2}, i_{7}\right\}\right\}$. Note the pattern instance $\left\{i_{4}, i_{8}\right\}$ is dropped from the table instance

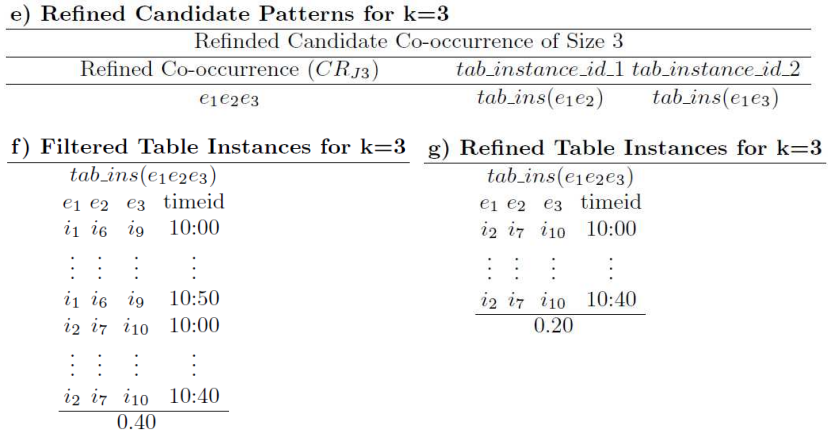

Figure 7: (e) Candidate Patterns of size-3 and (f) Filtered $\left(T_{O M A X 3}\right)$ and $(g)$ Refined $\left(T R_{J 3}\right)$ Table Instances of size-3.

tab_ins $\left(e_{1} e_{2}\right)$ as it does not satisfy $c c e_{t h}=0.01$ (see Fig. 6 (c) and (d) to compare).

As another example, in Fig. $7(\mathrm{~g})$ we show the refined table instances generated from refined prevalent patterns obtained in step 6 for a $p i_{t h}=0.40$. In the table instance tab_ins $\left(e_{1} e_{2} e_{3}\right)$ shown in Fig. $7(\mathrm{~g})$, we show the key columns of pattern instances that satisfy the threshold $c c e_{t h}$ value calculated using $J$. Please note the pattern instance $\left\{i_{1}, i_{6}, i_{9}\right\}$ is dropped from the table instance tab_ins $\left(e_{1} e_{2} e_{3}\right)$ as its cce is smaller than our $c c e_{t h}=0.01$ (see Fig. 7 (f) and (g) to compare).

Generation of refined prevalent patterns size$(\boldsymbol{k}+\mathbf{1})$ (step 8). This function discovers refined size$(k+1)$ prevalent STCOPs by pruning $P_{O M A X(k+1)}$ that have $p i<p i_{t h}$. As seen from the Fig. $7(\mathrm{~g})$, the pattern $S E_{i}=\left\{e_{1}, e_{2}, e_{3}\right\}$ will be pruned if $p i_{t h}$ is set to 0.40 .

In step 9, we calculate the union of refined prevalent patterns. The algorithm runs iteratively until no more STCOPs can be generated (our $P R_{J(k+1)}$ is empty), and returns all prevalent STCOPs, in step 12. Since we do not have any 
patterns left that satisfies the threshold $p i_{t h}=0.40$ in our example data set shown in Fig. 2 and Table 1, the algorithm would terminate at $k=3$ for our running example.

\section{Experimental Evaluation}

In this section, we compare our FastSTCOPs-Miner algorithm against the classic Apriori-based approach [17] which we will call Naïve STCOPs algorithm. In our experiments, we are using three real-life data sets from the solar physics domain and one artificial data set.

In the real-life data sets, we evaluate our algorithms using six types of evolving solar phenomena. Our real-life data sets contain evolving instances of six different solar event types, which were observed on 01/01/2012 (denoted Data Set $A$ ), 01/01/2012 through 01/03/2012 (denoted Data Set $B$ ), and 01/01/2012 through 01/05/2012 (denoted Data Set $C)$. We obtained our data sets from the well-known solar data repository called Heliophysics Event Knowledgebase (HEK) [7],[19]. The six different solar event types in our data sets are: Active Region, Filament, Sigmoid, Sunspot, Flare, and Emerging Flux.

The artificial data set (denoted Data Set D) is generated based on the works of Huang et al. in [9]. The artificial data set generator creates a data set of event instances with spatiotemporal features for spatial framework of size $D \times D$. Event types are generated with random size, speed, duration and area change parameters. Number of events to be generated is an input parameter to dataset generator, $M$. We used artificial data set to investigate behaviour of algorithms for larger number of event types. All of our data sets are available on-line to let researchers interested in this topic reproduce our experiments, and maybe even improve on our solution. The website for this paper/research can be found at [8].

We investigated the FastSTCOPs-Miner algorithm and Naïve STCOPs algorithm to accurately capture the STCOPs of the six different solar event types in the reallife data sets, and nine different artificial event types in the artificial data set. In all four data sets instances of different event types are represented as evolving polygons, where each instance of these events has significantly different spatial size, duration of life time and dynamics of its evolution. We compare and report the number of pattern instances found, the execution time of the algorithms, and the storage space requirements of the algorithms. For the three real-life data sets, for both algorithms, the $c c e_{t h}$ values were set to $0.01, p i_{t h}$ values were set to 0.1 , and the sampling time interval $\Delta t$ was set at 30 minutes leading to exactly the same set of final STCOPs. For the artificial data set, for both algorithms, the $c c e_{t h}$ values were set to $0.01, p i_{t h}$ values were set to 0.05 , and the sampling time interval $\Delta t$ were set at 3 minutes. All experiments were performed using PostgreSQL 9.1.4 and PostGIS 1.5.4. We report results highlighting memory usage efficiency and execution time of our FastSTCOPs-Miner algorithm in comparison to the Naïve STCOPs algorithm.

\subsection{Memory Usage}

We first investigated the memory usage of the FastSTCOPsMiner and Naïve STCOPs algorithms for the candidate table instances generated. We report the hard-drive memory usage of candidate table instances with all the pattern instances generated (see bars in solid colors - black and white in Fig. 8), and memory usage of candidate table instances af-

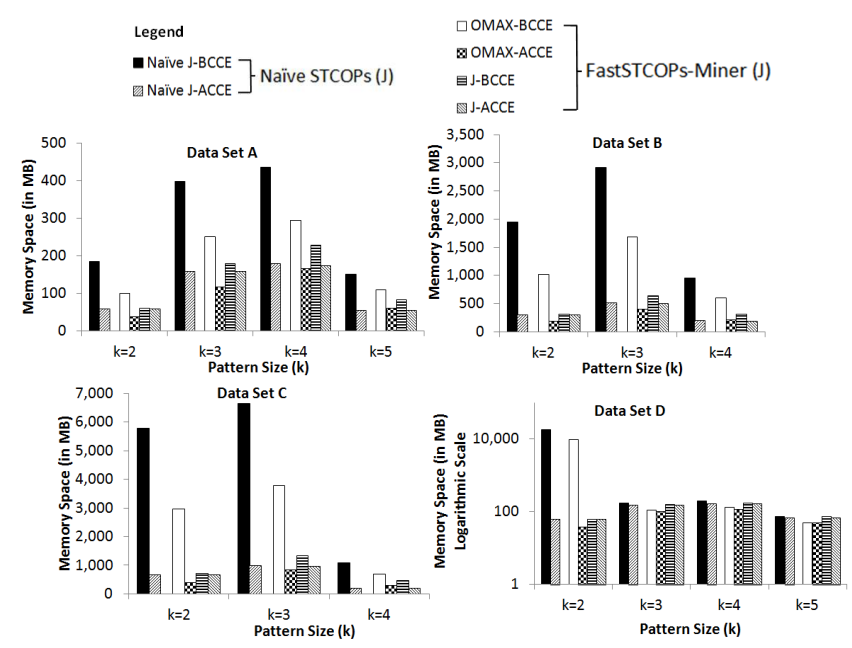

Figure 8: Memory usage used by candidate table instances for the FastSTCOPs-Miner and Nä̈ve STCOPs algorithms.

ter filtering the pattern instances that do not satisfy threshold $c_{\text {th }}$ (see bars with pattern markings in Fig. 8). In Fig. 8 bar labelled Naïve J-BCCE (black bars) represents the memory usage of table instances for all the pattern instances generated (i.e. the candidates), and Naïve J-ACCE (bars with diagonal upward stripes) represents the memory usage of table instances after pruning out the pattern instances that do not satisfy the threshold $c c e_{t h}$ set up for our experiments (i.e. our actual patterns that are reported on the output). In other words, J-BCCE compared to JACCE can be interpretted as candidates to actual patterns ratio in classical Apriori and are indicators of measures selectivity. As we can see from the first two bars in the first chart shown in Fig. 8 Jaccard measure was heavily used to prune the $187 \mathrm{MB}$ of candidates for pattern instances to $50 \mathrm{MB}$ of actual size-2 pattern instances. This is what has happened in the Naïve STCOPs algorithm. The next four bars in each chart shown in Fig. 8 show effectiveness of our FastSTCOPs-Miner algorithm. The first of these four bars (labelled OMAX-BCCE, in white solid color) represents candidate pattern instances that we are about to filter using $O M A X$ measure. As we can see this amount of memory is already smaller than storage needed for candidates (com-

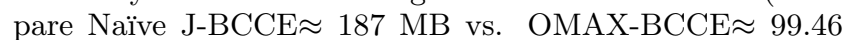
$\mathrm{MB}$ in Fig. 8 Data Set A). This is because of the fact that to accomplish pruning using Jaccard measure we have to precompute and store both Union and Intersection volumes for the co-occurring patterns, while when we use $O M A X$ only Intersection volumes are needed. This is what causes the reduction in storage in the FastSTCOPs-Miner algorithm (from $187 \mathrm{MB}$ in the first black/Naïve J-BCCE bar to 99.46 MB in the first white/OMAX-BCCE bar). This benefit continues through remaining steps of our algorithm. As expected, from Fig. 8 we can observe, that there is a drop in the memory usage after the pattern instances are pruned out by applying the threshold $c c e_{t h}$ (see and compare Naïve J-BCCE in black color with Naïve J-ACCE marked with diagonal upward stripes in Fig. 8). However, generation of all J-BCCE's is neccessary to discover actual STCOPs (i.e our J-ACCE's).

Also, in Fig. 8 OMAX-BCCE represents the memory usage of table instances for all the pattern instances generated. OMAX-ACCE represents the memory usage of ta- 
ble instances after filtering the pattern instances that do not satisfy the threshold $c c e_{t h}$ in FastSTCOPs-Miner algorithm. This time OMAX-BCCE to OMAX-ACCE ratio represents selectivity (i.e. pruning power) of our filter step in the FastSTCOPs-Miner algorithm. Please note, here the cce value is calculated using $O M A X$, so the $J$ based refine step is fed by the filtered out data (i.e. satisfying $c c e_{t h}$ and $\left.p i_{t h}\left(p i_{O M A X}\right)\right)$ but without missing any relevant patterns. Moreover, J-BCCE represents the memory usage of table instances for all the pattern instances generated from the filtered pattern instances (that is from our OMAX-ACCE bars that satisfy $\left.p i_{t h}\left(p i_{O M A X}\right)\right)$ and JACCE represents the memory usage of table instances after filtering the pattern instances that do not satisfy the threshold $\mathrm{cce}_{t h}$ in FastSTCOPs-Miner algorithm. Please note, here the cce value is calculated using $J$, to find patterns that are relevant. Also, the total number of pattern instances for OMAX-ACCE (bars with checkered pattern) is greater than or equal to J-BCCE (because of filtering effect of threshold $\left.p i_{t h}\left(p i_{O M A X}\right)\right)$; however, the memory usage increases for J-BCCE because of the union geometries generated for all of the pattern instances in order to calculate cce's using $J$ measure. Furthermore, from Fig. 8 we can observe a decrease in the memory usage after the pattern instances are filtered by applying threshold cce $_{t h}$ (please compare OMAX-BCCE with OMAX-ACCE and J-BCCE with J-ACCE, respectively). This shows the effectiveness of the anti-monotone property of the measures $J$ and $O M A X$ (see Lemma 3.1 and 3.2) and the benefit of our $O M A X$-based pruning strategy (see Lemma 3.4).

\subsection{Execution Time}

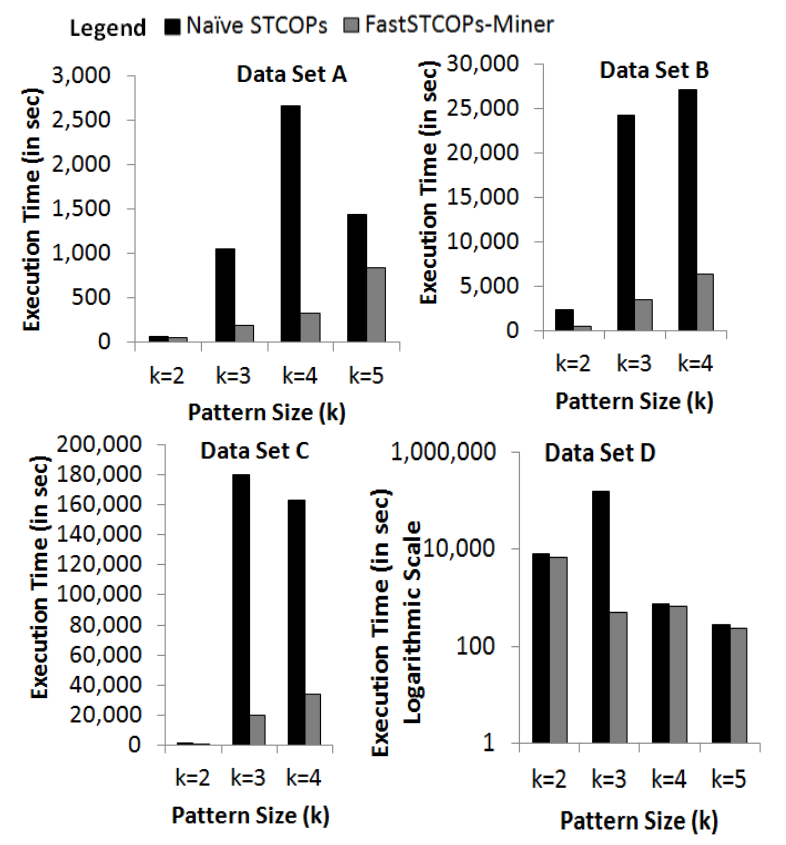

Figure 9: Camparison of execution time for the FastSTCOPsMiner and Nä̈ve STCOPs.

Next, we show the execution times of our FastSTCOPsMiner and Naïve STCOPs algorithms. Fig. 9 shows the execution time for patterns of different sizes. As expected, our FastSTCOPs-Miner algorithm outperforms the original Naïve STCOPs algorithm, since it uses a filter-and-refine strategy to find pattern instances that satisfy the threshold $c c e_{t h}$ for $J$. The Naïve STCOPs algorithm generates computationally expensive Union geometries for all the pattern instances (see the bars labelled as Naïve J-BCCE in Fig. 8 to realize how much memory overhead this process generates for data sets $A, B, C$, and $D$ ), while our FastSTCOPs-Miner algorithm generates Union geometries for smaller data set (see the bars labelled as J-BCCE in Fig. 8). This memory overhead causes the execution time of the Naïve STCOPs algorithm to be slower in comparison to our FastSTCOPsMiner algorithm.

\subsection{Pattern Instances}

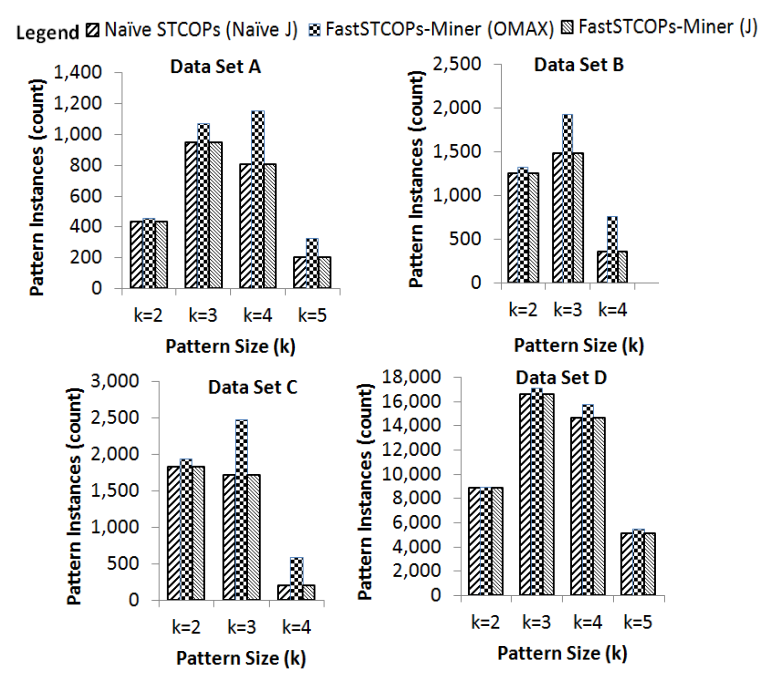

Figure 10: Pattern instances found (filtered with OMAX and refined with J) using the FastSTCOPs-Miner and Naïve STCOPs.

Fig. 10 shows the counts of pattern instances that satisfy the threshold value $c c e_{t h}$. We compare the counts of pattern instances satisfying the threshold cce $_{t h}$ with $O M A X$ (FastSTCOPs-Miner (OMAX)) and $J$ (FastSTCOPs-Miner $(\mathrm{J})$ ) for our FastSTCOPs-Miner algorithm. We also compared the counts of pattern instances of the FastSTCOPsMiner and Naïve STCOPs algorithms. As shown in Lemma 3.3, we can observe in Fig. 10 that the selectivity of the measures $O M A X$ and $J$ follows the order $O M A X \geq J$. Moreover, the count of pattern instances found for the threshold value $c c e_{t h}$ is the same for our FastSTCOPs-Miner $(J)$ and the Naïve STCOPs $(J)$ algorithm in Fig. 10. The identical results between FastSTCOPs-Miner $(J)$ and Naïve STCOPs $(J)$ confirm the correctness of our implementation of the FastSTCOPs-Miner algorithm.

\section{Conclusion and Future Work}

Empirical results on three real-life data sets, drawn from the solar physics discipline, and an artificial data set, serve to validate our FastSTCOPs-Miner algorithm. From the result of Fig. 8, we exhibit the memory space usage of candidate table instances for the FastSTCOPs-Miner and the Naïve STCOPs algorithms by showing the memory space comparison before and after pruning the pattern instances with threshold $c c e_{t h}$. This result is important in order to see the effects of the anti-monotonic property of the measures $J$ and $O M A X$ (see Lemma 3.1 and 3.2). We also showed that our FastSTCOPs-Miner algorithm is faster in comparison to Naïve STCOPs algorithm in Fig. 9. These results shows the significance of our filter and refine approach used in FastSTCOPs-Miner algorithm letting us to create 
the fastest STCOPs mining algorithm from evolving regions that is currently available on our planet.

For the future work, we plan to investigate developing non-Apriori approaches to discover STCOPs. One approach is to use frequent pattern growth [6] technique to discover STCOPs. Frequent pattern growth approach is shown to outperform Apriori-based approaches [6].

\section{Acknowledgment}

This work was supported by two National Aeronautics and Space Administration (NASA) grant awards, 1) No. NNX09AB03G and 2) No. NNX11AM13A.

\section{References}

[1] R. Agrawal, T. Imieliński, and A. Swami. Mining association rules between sets of items in large databases. SIGMOD Rec., 22(2):207-216, June 1993.

[2] J. Becla1a, A. Hanushevskya, S. Nikolaevb, G. Abdullab, A. Szalayc, M. Nieto-Santistebanc, A. Thakarc, and J. Grayd. Designing a multi-petabyte database for LSST. In Proc. of SPIE 6270, Observatory Operations: Strategies, Processes, and Systems, 62700R , 2006.

[3] K. D. Borne. Astroinformatics: A 21st Century Approach to Astronomy, 2009.

[4] H. Cao, N. Mamoulis, and D. W. Cheung. Discovery of collocation episodes in spatiotemporal data. In Proc. of the Sixth International Conference on Data Mining, ICDM '06, pages 823-827, Washington, DC, USA, 2006. IEEE Computer Society.

[5] M. Celik, S. Shekhar, J. P. Rogers, J. A. Shine, and J. S. Yoo. Mixed-drove spatio-temporal co-occurence pattern mining: A summary of results. In Proc. of the Sixth International Conference on Data Mining, ICDM '06, pages 119-128, Washington, DC, USA, 2006. IEEE Computer Society.

[6] J. Han and J. Pei. Mining frequent patterns by pattern-growth: methodology and implications. SIGKDD Explor. Newsl., 2(2):14-20, Dec. 2000.

[7] HEK. http://www.lmsal.com/isolsearch, Jan 2012.

[8] STCOPs. https://www.cs.montana.edu/ k.ganesanpillai/, June 2013.

[9] Y. Huang, S. Shekhar, and H. Xiong. Discovering colocation patterns from spatial data sets: a general approach. IEEE Transactions on Knowledge and Data Engineering, 16(12):1472-1485, 2004.

[10] K. Lang. The Cambridge Encyclopedia of the Sun. Cambridge University Press, 2001.

[11] S. Langhof and T. Straume. Workshop Report on Space Weather Risks and Society In Report of a workshop sponsored by and held at NASA Ames Research Center, 2011.

[12] C. D. Manning and H. Schütze. Foundations of statistical natural language processing. MIT Press, Cambridge, MA, USA, 1999.

[13] P. Martens, G. Attrill, A. Davey, A. Engell, S. Farid, P. Grigis, J. Kasper, K. Korreck, S. Saar, A. Savcheva, Y. Su, P. T. M. Wills-Davey, P. Bernasconi, N.-E. Raouafi, V. Delouille, J. Hochedez, J. Cirtain, C. DeForest, R. Angryk, I. D. Moortel, T. Wiegelmann, M. Georgoulis, R. McAteer, and
R. Timmons. Computer Vision for the Solar Dynamics Observatory (SDO), 2010.

[14] E. R. Omiecinski. Alternative interest measures for mining associations in databases. IEEE Trans. on Knowl. and Data Eng., 15(1):57-69, Jan. 2003.

[15] D. Patel. Interval-orientation patterns in spatio-temporal databases. In Database and Expert Systems Applications, 6261:416-431, 2010.

[16] W. D. Pesnell, B. Thompson, and P. Chamberlin. The Solar Dynamics Observatory (SDO). Solar Physics, 275(1-2):3-15, 2012.

[17] K. G. Pillai, R. A. Angryk, J. M. Banda, M. A. Schuh, and T. Wylie. Spatio-temporal co-occurrence pattern mining in data sets with evolving regions. In ICDM Workshops, pages 805-812, 2012.

[18] K. G. Pillai, R. A. Angryk, J. M. Banda, T. Wylie, and M. A. Schuh. Spatiotemporal co-occurrence rules. In New Trends in Databases and Information Systems. Springer, 241:27-35, 2013.

[19] M. A. Schuh, R. A. Angryk, K. G. Pillai, J. M. Banda, and P. C. Martens. A large-scale solar image dataset with labeled event regions. In Int. Conf. on Image Processing (ICIP), 2013.

[20] P.-N. Tan, M. Steinbach, and V. Kumar. Introduction to Data Mining, (First Edition). Addison-Wesley Longman Publishing Co., Inc., Boston, MA, USA, 2005.

[21] P. Taylor. Quantitative Methods in Geography: An Introduction to Spatial Analysis. Houghton Mifflin, 1977.

[22] J. Wang, W. Hsu, and M. L. Lee. A framework for mining topological patterns in spatio-temporal databases. In Proc. of the 14th ACM international conference on Information and knowledge management, CIKM '05, pages 429-436, New York, NY, USA, 2005. ACM.

[23] H. Xiong, S. Shekhar, Y. Huang, V. Kumar, X. Ma, and J. S. Yoo. A framework for discovering co-location patterns in data sets with extended spatial objects. In SDM, 2004.

[24] H. Yang, S. Parthasarathy, and S. Mehta. A generalized framework for mining spatio-temporal patterns in scientific data. In Patterns in Scientific Data, ACM SIGKDD Int'l Conf. on Knowledge Discovery and Data Mining, pages 716-721, 2005. 